\title{
Mediterranean diet and metabolic syndrome in three countries of Calabria
}

\author{
Cornelli $\mathrm{U}^{1 *}$, Cassano $\mathrm{G}^{2}$, Meringolo $\mathrm{G}^{3}$, Rausa $\mathrm{M}^{4}$, Valente $\mathrm{O}^{5}$ and Recchia $\mathrm{M}^{6}$ \\ ${ }^{1}$ Loyola University School of Medicine, Chicago, USA \\ ${ }^{2}$ MAP (Monitoring Alimentary Pathology center, Rende, Italy \\ ${ }^{3}$ Department of Cardiology, Rende Hospital, Italy \\ ${ }^{4}$ Department of Public Health, Rende, Italy \\ ${ }^{5}$ Municipal administration, Rovito, Italy \\ ${ }^{6}$ Statistical Dept University of Lugano, Switzerland
}

\begin{abstract}
Mediterranean diet has been associated with low prevalence and progression of metabolic syndrome (MetS). However, no specific foods have been described as causative of MetS.

Six hundred subjects of both sexes (between 35-65 years) have been studied in the investigational centers of MAP (Monitoraggio Alimenti Patologia) organized by the local Municipalities of three countries in Calabria-Italy. The enrollment was suggested by family doctors who were addressing subjects suffering from MetS matched with subject non MetS in the same age range. Food intake was analyzed through a weakly questionnaire (FIA or Food Intake assessment).

529 subjects ( $85 \mathrm{MetS} / 297$ females, and $75 \mathrm{MetS} / 232$ males) concluded the study. In both genders cardiovascular diseases and ultrasound carotid damages were significantly higher, and in males osteoarthosis also was more frequent. Caloric intake was significantly more consistent in MetS with no distinction among carbohydrates, proteins, fibers or fats but with different food pattern in females and males.

Among 77 different foods, in females more white meat, less carrots, cauliflower and red wine were found in MetS. In males red wine, beer, garlic, milk, salami, fruit of the season, and chocolate were lower in MetS and canned tuna was higher.

Multivariate analysis indicate that a complex food combination is determining MetS, with different figures for males and females.

In an area typical for the Mediterranean diet, MetS seems determined by an excessive caloric intake with different food pattern depending upon the gender, indicating that it is not possible to generalize about diet components as causative of the disease.
\end{abstract}

\section{Introduction}

Metabolic syndrome (MetS) is a multi-factorial disorder including hyperglycemia, dyslipidemia, hypertension, and abdominal obesity that is becoming one of the most common pathologies affecting the adult worldwide in a similar proportion for both genders [1,2].

Despite some argument about its role and value in clinical practice, subjects presenting the three of the 5 components as defined by the ATP III (Adult Panel Treatment III) consisting of the increase of blood glucose, triglycerides, blood pressure, abdominal circumference, and reduction of HDL cholesterol, have five times risk or more for type 2 diabetes mellitus, three times risk of developing coronary heart attack or stroke, and two times higher cardiovascular mortality than subjects without the syndrome $[1,3]$.

Considering the pathophysiology, overweight and obesity are central to the risk of the disease predisposing to the insulin resistance, hypertension and dyslipidemia.

Evidence of genetic component have been suggested in term of heritability: family studies provided information about a consistent heritability of some component; linkage studies have shown combinations among the different determinants (e.g, HDL, blood pressure, triglyceride, abdominal visceral fats) in Caucasian, AfricanAmerican, Mexican-American; genoma wide association studies (GWAS) have detected some single nucleotide polymorphism of fat mass and obesity associated protein (FTO) [4-8].

The inflammatory condition and modification of the gut microbiota are also considered as causative and witness the complexity of the disease $[9,10]$.

The prevalence of MetS is increasing despite a large difference between countries estimated from 10 to $84 \%$ depending on the ethnicity, age, gender, and race of the population [11]. Accompanied to a rapid increase of the life expectancy, it is estimated that around a quarter of the world's adult population has MetS.

${ }^{\star}$ Correspondence to: Umberto Cornelli, Loyola University School of Medicine, Chicago, 2160 First Ave Maywood IL, Piazza Novelli 520129 Milan, Italy, E-mail: ucornelli@gmail.com

Key words: mediterranean diet, metabolic syndrome, calabria

Received: November 20, 2018; Accepted: December 03, 2018; Published: December 06, 2018 
The major risk factor for developing the disease are considered to be the diet rich in fats and carbohydrates and physical inactivity [1,2].

However, among carbohydrates and lipids many distinctions have been made.

For carbohydrates, although they are the only foods constituents that directly increase blood glucose, a distinction has to be made between those with high glycemic index (GI) or glycemic load (GL) -as refined grains, potatoes and sugar sweetened beverages- from other foods as the minimally processed grains, legumes, whole fruits and also pasta in that they do not have a sudden impact on insulin release because of the hifgh fiber content [12]. At the end the quality of carbohydrates seems to have a more important role than the quantity.

The same is for lipids, because saturated fats have different metabolic activity compared to unsaturated lipids which behave differently depending upon the degree of unsaturation as for seed oil, extravirgin olive oil, palm oil. Even more the production process to refine oils can generate toxic compounds as for palm oil.

Lifestyle modification is considered one of the keys to counteract the MetS.

In a meta-analysis of some randomized controlled study on lifestyle modification was shown that the adherence to a healthy diet or a combination of diet and physical activity increase were associated with the reversion of MetS [13]. Talking about healthy diet, in a meta-analysis of several studies (50) -consisting in total of $>500.000$ subjects- the Mediterranean diet was shown to be appropriate by all the population groups for primary and secondary prevention of the disease $[14,15]$.

Italy, Spain, Serbia, Greece -just to mention some of the countries on the Mediterranean Sea- use to eat very different foods, or similar foods with different recipe. Despite the common use of extra virgin olive oil, fruit and vegetables in this diet one may consider also that countries are not comparable in terms of MetS prevention or treatment due to environment and lifestyle differences.

The consequence of all these distinctions is that the total amount of food components in the diet such as carbohydrates, lipids, proteins, fibers do not give enough information on the MetS development.

Even more, the same components in different area of the same country may have a different impact. In other terms, is almost impossible to draw conclusion about the effect of foods in determining the MetS unless a restricted territory will be analyzed where subjects are used to eat similar food and similar traditional way of preparing foods.

For this reason, after having crossed the Italy with a mobile unit [16] we decided to choose an Italian region (Calabria) where to start an observational study in different towns controlling the food intake in subjects with MetS compared to matched non MetS people. The aim was to identify what kind of food if any was relating to the disease using a weekly validated Food Intake questionnaire (FIA) consisting of 250 different foods [17].

\section{Material and methods}

This research represents a pilot study of the three countries aimed to drive the long-term study (10 years) that will consider the relationship between Foods and MetS (called MAP or Monitoraggio Alimenti Patologia) in ten countries of Calabria-Italy. The data concerning the first evaluation of 600 cases were evaluated.

Three countries were selected: Rovito which is located on a hill, San Lucido on the sea side, and Rende on the plain.

\section{Enrollment method}

A conference on "food and pathology" open to the public was organized in three towns (Rovito, San Lucido, and Rende) with the aid of the relative Municipalities. Family doctors were asked to participate and requested to help in the enrollment of their patients suffering from MetS matched with other non-MetS subjects coming for a routine check out.

The enrolment duration was three month/town in the same period of the year and in total the study lasted 3 years. The Ethical Committees of the three Municipalities involved approved the protocol.

\section{Admission criteria}

Subject of both sexes aging between 35 and 60 years were admitted suffering from MetS matched with subjects suffering from other diseases provided that only one of the 5 typical variables of MetS (according to ATP III or Adult Treatment Panel) was present. The capacity to fill up correctly the FIA (weakly Food Intake Assessment-see later) after the training was a prerequisite to enter the study.

\section{Exclusion criteria}

Cancer of any type, severe psychiatric or metabolic disorders, chronic diseases not under adequate therapy control, more than one variable of MetS according to ATP III, FIA questionnaire unreliable.

\section{FIA questionnaire}

FIA consisted of a list of the most common 250 foods in Italy [17]. Following a training with a nutritionist the subject had to fill up a 7 days questionnaire recording the amount (in $\mathrm{g}$ or $\mathrm{mL}$ ) of each of the listed foods. The questionnaire was fundamental for the study, and subjects presenting records with a caloric intake lower than $90 \%$ of the Mifflin St Jeor (MSJ) were excluded. However, the latter were excluded from the current evaluation but continued study.

The FIA was taken at least twice in the period of two years of observation and the data were reported as averages.

Smokers were included in the study and were considered as current smokers or nonsmokers. The latter where those subjects with at least 5 years of interruption. Physical activity was measured through a very simple 4 points questionnaire considering the following items: sedentariness, limited activity, normal activity, physical training.

\section{Lab analysis and anthropometric measures, echography}

The common variables for MetS according to ATP III were measured consisting of HDL, triglycerides (TG), blood pressure (BP), abdominal circumference $(\mathrm{AC})$, and glucose. Blood samples were taken before the enrollment to confirm the diagnosis.

The enrolled subjects underwent to the ultrasound carotid analysis using VIVID L8 ultrasound machine. The degree of the arterial lesion was according to a classification into VI different classes: from Class I (normal artery) to class VI (presence of complex plaques with stenosis) [18].

\section{Statistical analysis}

\section{Sample}

The number of cases to be enrolled was on heuristic base to analyzes the possibility continue the long-term study and in case to adapt the protocol. Two hundred cases for each country (600 cases in total) were analyzed considering obtaining valuable data in at least 500 cases. With these figures, the hypothesis was to analyze about 30 
$\%$ of the cases with a MetS. However, to be sure to obtain at least 150 cases of MetS, the family doctors were asked to make a first selection consisting of one case of MetS (no matter about the sex) and two matched patients non MetS provided within the admission criteria.

\section{Calculations}

Each variable was analyzed calculating the average and SD. The $t$ test or Wilcoxon test were used to determine the relative differences between groups (MetS, Vs non MetS defined as "Controls") for all the variables. The frequencies of concomitant diseases in the two groups were measured using the chi square test (Fisher, with or without Yates correction).

For what concern the FIA, the average weekly data (of two FIA taken within the period) for any food (e.g. pasta, wine, red meat) were determined only to have a rough indication of the quantities (in $g$ or $\mathrm{mL}$ ). Following the control of the variables distribution, the category analysis was based on discretization (D) of each variable using from 2 to 5 (or D2-D5) different cut off. The Pearson chi square was used to differentiate the two groups followed by the Nominal Logistic Fit value.

For each food, odds ratios were analyzed to compare the differences between various $\mathrm{D}$ levels comparing controls and MetS. The logistic regression model (Likelihood Ratio test) was applied to analyze the connection between foods within MetS and controls to describe which were more frequent in MetS compared to controls [19].

\section{Results}

In the three selected countries Rovito, San Lucido and Rende a total of 600 subjects were analyzed.

The flow chart of the study is reported in Table 1.

The percentages of MetS in the three towns were respectively 32.6 \% in Rovito, $31.6 \%$ in San Lucido, and $26.7 \%$ in Rende. These values indicate that the family doctors were making a similar selection of the cases.

The compliance of the study was $88 \%$ since only 529 subjects concluded correctly the questionnaire. Seventy-one were excluded: 47 because of an incorrect FIA, and 24 were not returning at all the FIA.

The general characteristics of the subjects are reported in Table 2. The caloric intake measured through FIA analysis was between the
$96 \%$ to $98 \%$ of the theoretical intake calculated according to the MSJ formula.

The differences between controls and MetS subjects were statistically significant $(t$ test $\mathrm{p}<0.05)$ for all the variables that are typical of the syndrome.

Total and LDL cholesterol in male with MetS was found lower than in controls despite the differences were not statistically significant $(t$ test $p>0.05$ ). This could be due to the treatment of dyslipidemia that was more common in these subjects than in control group (respectively 9.3\% Vs $5.1 \%$ : see Table 3).

The total caloric intake measured according to FIA was significantly higher in both groups with MetS and similar to the respective MSJ formula (the differences were $<4 \%$ ).

For what concerns the concomitant diseases, in the group of MetS the number of subjects suffering from cardiovascular disease was significantly higher (chi square $\mathrm{p}<0.01$ ) in both males and females (Table 3). This was confirmed by the ultrasound analysis (ECO) where class IV images (presence of small plaques) were higher in both groups of MetS although the difference was significant in males only (chi square test $\mathrm{p}<0.05$ ). The percentages of all the other diseases were similar, a part of osteroarthrosis that was present with significantly higher incidence in males only.

In males a higher incidence of total diseases/person in the groups of MetS was found, the differences were statistically significant (Wilcoxon test $\mathrm{p}<0.05$ ). In females the total disease/person were slowly higher $(+12.4 \%)$ but not statistically significant (Wilcoxon test $\mathrm{p}>0.05)$.

Considering the main food components, the only difference between groups was detected for proteins intake with a significant increase in males with MetS, and surprisingly a lower intake of alcohol (mainly due to lower red wine drinking) was shown (Table 4). Carbohydrates and lipids do not present any important difference between groups as for the fibers. The ratio carbohydrates/lipids or soluble sugars/lipids in term of caloric intake were not significantly different in both sexes.

Table 1. Enrollment flow chart

\begin{tabular}{|c|c|c|c|c|}
\hline & \multicolumn{3}{|c|}{ Towns } & \multirow{2}{*}{ Total } \\
\hline & Rovito & San Lucido & Rende & \\
\hline Subjects & 200 & 200 & 200 & 600 \\
\hline Drop out (no FIA report) & 3 & 15 & 6 & 24 \\
\hline FIA Incorrect & 13 & 27 & 7 & 47 \\
\hline Total non-included & 16 & 42 & 13 & 71 \\
\hline Females Controls & 72 & 69 & 71 & 212 \\
\hline Females with MetS & 30 & 38 & 17 & 85 \\
\hline Male controls & 52 & 39 & 66 & 157 \\
\hline Male with MetS & 30 & 12 & 33 & 75 \\
\hline Total females & 102 & 107 & 88 & 297 \\
\hline Total males & 82 & 51 & 99 & 232 \\
\hline Total evaluated & 184 & 158 & 187 & 529 \\
\hline
\end{tabular}


Table 2. General characteristics of the subjects: mean values \pm SD or frequencies

\begin{tabular}{|c|c|c|c|c|c|c|c|c|c|}
\hline Gender & & & & & & & & & \\
\hline $\begin{array}{l}\text { Disease } \\
{[\mathrm{N} \text { of cases }]}\end{array}$ & & $\begin{array}{c}\text { Controls } \\
\text { [212] }\end{array}$ & & $\begin{array}{c}\text { MetS } \\
{[85]}\end{array}$ & & $\begin{array}{c}\text { Controls } \\
\text { [157] }\end{array}$ & & $\begin{array}{r}\text { MetS } \\
{[75]}\end{array}$ & \\
\hline Variables & Measure & Mean & SD & Mean & SD & Mean & SD & Mean & $\mathrm{SD}$ \\
\hline Age & Years & 47.5 & 6.95 & 47.5 & 7.97 & 50.0 & 7.98 & 51.9 & 8.35 \\
\hline Height & $\mathrm{m}$ & 1.58 & 0.055 & 1.59 & 0.054 & 1.70 & 0.061 & 1.71 & 0.072 \\
\hline Weight & $\mathrm{Kg}$ & 66.1 & 11.26 & $79.9^{\mathrm{a}}$ & 15.7 & 80.2 & 11.59 & $89.9^{\mathrm{a}}$ & 11.70 \\
\hline BMI & $\mathrm{Kg} / \mathrm{m}^{2}$ & 26.6 & 4.69 & $31.7^{\mathrm{a}}$ & 5.37 & 27.9 & 3.26 & $30.7^{\mathrm{a}}$ & 3.87 \\
\hline $\mathrm{AC}$ & $\mathrm{cm}$ & 87.9 & 13.93 & $102.4^{\mathrm{a}}$ & 19.42 & 97.1 & 13.68 & $105.4^{\mathrm{a}}$ & 25.45 \\
\hline BP min & $\mathrm{mmHg}$ & 82 & 27.0 & $87^{\mathrm{a}}$ & 28.3 & 82 & 28.3 & $88^{\mathrm{a}}$ & 35.9 \\
\hline BP mx & $\mathrm{mmHg}$ & 129 & 47.8 & $139^{\mathrm{a}}$ & 46.7 & 132 & 50.4 & $140^{\mathrm{a}}$ & 49.8 \\
\hline Total CH & $\mathrm{mg} / \mathrm{dL}$ & 205 & 37.9 & 212 & 41.6 & 214 & 37.7 & 204 & 36.6 \\
\hline LDL & $\mathrm{mg} / \mathrm{dL}$ & 126 & 33.2 & $138^{\mathrm{a}}$ & 40.4 & 137 & 34.6 & 130 & 36.4 \\
\hline HDL & $\mathrm{mg} / \mathrm{dL}$ & 61 & 11.5 & $46^{\mathrm{a}}$ & 11.1 & 51 & 9.9 & $40^{\mathrm{a}}$ & 11.2 \\
\hline Triglycerides & $\mathrm{mg} / \mathrm{dL}$ & 101 & 34.5 & $175^{\mathrm{a}}$ & 63.5 & 134 & 57.8 & $245^{\mathrm{a}}$ & 153.3 \\
\hline Glucose & $\mathrm{mg} / \mathrm{dL}$ & 88 & 10.8 & $94^{\mathrm{a}}$ & 16.8 & 93 & 13.6 & $103^{\mathrm{a}}$ & 29.0 \\
\hline Smoking & Yes/no & $12 / 200$ & & $5 / 85$ & & $8 / 149$ & & $5 / 70$ & \\
\hline Physical activity ${ }^{\mathrm{b}}$ & $\mathrm{S} / \mathrm{O}$ & $195 / 17$ & & $77 / 8$ & & $150 / 7$ & & $8 / 69$ & \\
\hline $\mathrm{MSJ}^{\mathrm{c}}$ & Kcal/week & 11751 & 1479.1 & 12923 & 1511.8 & 15437 & 1659.2 & 16136 & 1882.0 \\
\hline Calorie intake $^{\mathrm{d}}$ & Kcal/week & 11304 & 4021.4 & 12378 & 4892.0 & 15045 & 5043.1 & 15752 & 4855.4 \\
\hline
\end{tabular}

${ }^{a}$ t test Controls Vs MetS $\mathrm{p}<0.05 ;{ }^{\mathrm{b}} \mathrm{S}=$ sedentariness and $\mathrm{O}=$ limited activity, normal activity, physical training; ${ }^{\mathrm{C}}$ Caloric intake using Mifflin St Jeor formula; ${ }^{\mathrm{d}} \mathrm{Caloric}$ intake calculated from the FIA

Table 3. Concomitant diseases: percentage of the total subjects

\begin{tabular}{|c|c|c|c|c|}
\hline \multirow{2}{*}{\begin{tabular}{|l} 
Gender \\
Disease \\
[N of cases]
\end{tabular}} & \multicolumn{2}{|c|}{ Females } & \multicolumn{2}{|c|}{ Males } \\
\hline & $\begin{array}{c}\text { Controls } \\
{[212]}\end{array}$ & $\begin{array}{l}\text { MetS } \\
{[85]}\end{array}$ & $\begin{array}{c}\text { Controls } \\
\text { [157] }\end{array}$ & $\begin{array}{c}\text { MetS } \\
{[75]}\end{array}$ \\
\hline $\begin{array}{l}\text { Concomitant } \\
\text { disease }\end{array}$ & $\%$ & $\%$ & $\%$ & $\%$ \\
\hline Allergic & 1.9 & 1.2 & 2.5 & 4.0 \\
\hline Bronchopulmonary & 0.5 & 0 & 0 & 0 \\
\hline Cardiovascular & 13.2 & $29.4^{\mathrm{a}}$ & 15.3 & $38.7^{\mathrm{a}}$ \\
\hline Dyslipidemic & 4.7 & 3.5 & 5.1 & 9.3 \\
\hline Dermatological & 0.5 & 0 & 0.5 & 0 \\
\hline Endocrinological & 10.4 & 10.5 & 1.3 & 2.7 \\
\hline ENT & 0.5 & 1.2 & 0 & 0 \\
\hline Gastroenterological & 3.3 & 7.1 & 2.5 & 8.0 \\
\hline Gynecological & 0.9 & 0 & - & - \\
\hline Hematological & 0.9 & 2.4 & 0 & 2.7 \\
\hline Osteoarthritic & 58.0 & 50.6 & 31.8 & $45.3^{\mathrm{a}}$ \\
\hline Odonatological & 1.4 & 1.2 & 0 & 2.7 \\
\hline Ophthalmological & 1.4 & 1.2 & 0 & 0 \\
\hline Neurological & 3.3 & 4.7 & 0.6 & 1.3 \\
\hline Psychiatric & 0.9 & 3.5 & 0 & 1.5 \\
\hline Urological & - & - & 3.2 & 6.7 \\
\hline Cancer $^{b}$ & 2.8 & 0 & 0 & 0 \\
\hline EC0 class IV & 5.7 & 11.8 & 3.8 & $14.7^{\mathrm{a}}$ \\
\hline Total/person & 1.10 & 1.24 & 0.6 & 1.30 \\
\hline$\%$ increase Vs control & & 13 & & 117 \\
\hline
\end{tabular}

${ }^{\text {a }}$ Chi square $\mathrm{p}<0.05$; ${ }^{\mathrm{b}}$ no malignancy

The analysis of food intake was conducted for all the 250 foods reported in the FIA (Food Intake Assessment). Seventy-seven type of food were measured (see Table 5) and the others up to 250 were not used or reported.

Following a careful analysis of foods distribution, for each item an arbitrary classification (discretization) was settled in terms of cut off forming from 2 to 5 different classes (Table 5).

Some of the listed foods in FIA were not part of the usual diet of the area or were used by few subjects only (e.g. mushrooms, pumpkin, pate, wurstel, big burghers, canned soup) and were not considered in the analysis. In total, the caloric intake of the foods listed accounted for at least the $90 \%$ of the total caloric amount/subject (Table 5).

The percentages of subjects within the given discretization were summarized together with the relative statistical analysis to compare controls and MetS subjects (Table 6).

The following results were found:

-for the carbohydrates-based foods, in females no differences were shown between controls and MetS, whereas in males the biscuits intake 
Table 4. Main food components as percentage of the total caloric intake and ratios with lipids: Mean \pm SD

\begin{tabular}{|c|c|c|c|c|c|c|c|c|}
\hline \multirow{3}{*}{\begin{tabular}{|l|} 
Gender \\
Disease \\
Components
\end{tabular}} & \multicolumn{4}{|c|}{ Females } & \multicolumn{4}{|c|}{ Males } \\
\hline & \multicolumn{2}{|c|}{ Controls } & \multicolumn{2}{|c|}{ MetS } & \multicolumn{2}{|c|}{ Controls } & \multicolumn{2}{|c|}{ MetS } \\
\hline & Mean & SD & Mean & SD & Mean & SD & Mean & SD \\
\hline Carbohydrates & 23.3 & 13.57 & 20.3 & 15.76 & 23.2 & 16.02 & 24.6 & 15.14 \\
\hline Soluble sugars & 26.3 & 12.72 & 29.3 & 17.16 & 25.1 & 15.28 & 24.7 & 13.53 \\
\hline Proteins & 16.3 & 2.86 & 16.8 & 4.25 & 16.0 & 2.96 & $16.7^{\mathrm{a}}$ & 2.26 \\
\hline Lipids & 33.8 & 5.98 & 33.6 & 6.90 & 31.0 & 6.13 & 31.4 & 6.28 \\
\hline Alcohol & 1.5 & 2.05 & 1.1 & 1.90 & 5.4 & 4.30 & $3.7^{\mathrm{a}}$ & 4.53 \\
\hline Fiber g/week & 93 & 43.8 & 95 & 61.1 & 102 & 42.8 & 98 & 52.6 \\
\hline Ratio carbohydrates/lipids & 0.72 & 0.469 & 0.62 & 0.517 & 0.78 & 0.585 & 0.88 & 0.758 \\
\hline $\begin{array}{l}\text { Ratio } \\
\text { soluble sugars/lipids }\end{array}$ & 0.82 & 0.526 & 0.91 & 0.560 & 0.86 & 0.630 & 0.82 & 0.484 \\
\hline
\end{tabular}

$\mathrm{a}=\mathrm{t}$ test Controls Vs MetS $\mathrm{p}<0.05$

Table 5. Average intake ( $\mathrm{g}$ or $\mathrm{mL}$ ) of different foods in Females (F) and Males (M) in the two groups of subjects, Controls and MetS, and relative discretization cut off for the different foods

\begin{tabular}{|c|c|c|c|c|c|c|c|c|}
\hline \multirow[t]{2}{*}{ Food } & \multirow{2}{*}{$\begin{array}{c}\begin{array}{c}\text { Controls } \\
\text { means }\end{array} \\
\mathrm{F} / \mathrm{M}\end{array}$} & \multirow{2}{*}{$\begin{array}{c}\begin{array}{c}\text { MetS } \\
\text { means }\end{array} \\
\text { F/M }\end{array}$} & \multicolumn{6}{|c|}{$\begin{array}{l}\text { Discretization categories } \\
\text { and cut off values }\end{array}$} \\
\hline & & & 0 & 1 & 2 & 3 & 4 & 5 \\
\hline \multicolumn{9}{|c|}{ Carbohydrates based food } \\
\hline Biscuits & $68 / 52$ & $54 / 40$ & 0 & 40 & 140 & 280 & 480 & - \\
\hline Bread & $433 / 587$ & $456 / 550$ & 0 & 200 & 450 & 950 & 2550 & - \\
\hline Breadsticks & $4 / 4$ & $8 / 2$ & 0 & 20 & 100 & 280 & - & - \\
\hline Croissant & $80 / 73$ & $80 / 68$ & 0 & 75 & 275 & 700 & - & - \\
\hline Crackers & $17 / 10$ & $22 / 7$ & 0 & 40 & 80 & 280 & - & - \\
\hline Gnocchi & $13 / 23$ & $15 / 14$ & 0 & 3 & 50 & 80 & - & - \\
\hline Oatmeal & $10 / 8$ & $15 / 8$ & 0 & 80 & 280 & 600 & - & - \\
\hline Rusks & $45 / 26$ & $62 / 26$ & 0 & 40 & 80 & 140 & 420 & - \\
\hline Sandwich homemade & $85 / 107$ & $55 / 97$ & 0 & 40 & 280 & 1120 & - & - \\
\hline Sandwich commercial & $13 / 22$ & $21 / 21$ & 0 & 54 & 315 & 630 & - & - \\
\hline Pasta & $304 / 404$ & $302 / 402$ & 0 & 182 & 395 & 690 & 1200 & - \\
\hline Polenta & $5 / 1$ & $7 / 3$ & 0 & 30 & 122 & 360 & - & - \\
\hline Pizza & $153 / 163$ & $153 / 140$ & 0 & 34 & 150 & 422 & 1470 & - \\
\hline Potatoes & $75 / 63$ & $42 / 63$ & 0 & 75 & 300 & 1060 & - & - \\
\hline Rice & $50 / 50$ & $55 / 49$ & 0 & 25 & 145 & 280 & 560 & - \\
\hline Tortellini & $11 / 13$ & $15 / 9$ & 0 & 25 & 100 & 240 & - & - \\
\hline \multicolumn{9}{|l|}{ Fruits } \\
\hline Apples & $476 / 560$ & $457 / 539$ & 0 & 200 & 700 & 1400 & 4200 & - \\
\hline Banana & $217 / 230$ & $189 / 171$ & 0 & 199 & 600 & 2800 & - & - \\
\hline Citrus fruit & $500 / 542$ & $360 / 498$ & 0 & 200 & 1700 & 4000 & - & - \\
\hline Dried fruit & $97 / 81$ & $73 / 114$ & 0 & 80 & 280 & 1900 & - & - \\
\hline Fruit in syrup & $8 / 20$ & $22 / 1$ & 0 & 18 & 100 & 1050 & - & - \\
\hline Fruit of the season ${ }^{b}$ & $460 / 521$ & $673 / 491$ & 0 & 475 & 950 & 1450 & 4000 & - \\
\hline Fruit Juice mL & $48 / 35$ & $65 / 104$ & 0 & 75 & 450 & 2100 & - & - \\
\hline Grapes & $65 / 43$ & $72 / 41$ & 0 & 50 & 300 & 2350 & - & - \\
\hline Homemade juice $\mathrm{mL}$ & $90 / 74$ & $85 / 130$ & 0 & 75 & 160 & 300 & - & - \\
\hline Pineapple & $30 / 18$ & $43 / 35$ & 0 & 68 & 300 & 495 & - & - \\
\hline Plums & $1 / 2$ & $1 / 0$ & 0 & 20 & 150 & 180 & - & - \\
\hline \multicolumn{9}{|l|}{ Vegetables/pulses } \\
\hline Carrots & $47 / 41$ & $36 / 33$ & 0 & 40 & 140 & 690 & - & - \\
\hline Cauliflower & $56 / 33$ & $40 / 35$ & 0 & 60 & 260 & 1820 & - & - \\
\hline Celery & $19 / 11$ & $21 / 12$ & 0 & 3 & 140 & 330 & - & - \\
\hline Chicory/lettuce & $187 / 193$ & $160 / 194$ & 0 & 90 & 245 & 490 & 500 & 1170 \\
\hline Fennels & $108 / 93$ & $70 / 124$ & 0 & 480 & 2000 & 3380 & - & - \\
\hline Garlic & $1.6 / 1.7$ & $1.7 / 1$ & 0 & 1.5 & 7 & 20 & - & - \\
\hline Onions & $82 / 83$ & $70 / 61$ & 0 & 90 & 290 & 630 & - & - \\
\hline Pepper & $61 / 68$ & $100 / 52$ & 0 & 50 & 250 & 1750 & - & - \\
\hline Pulses dry & $21 / 27$ & $21 / 25$ & 0 & 45 & 90 & 225 & - & - \\
\hline Pulses canned & $36 / 37$ & $33 / 33$ & 0 & 19 & 160 & 240 & 400 & - \\
\hline Savoy cabbage & $40 / 43$ & $50 / 45$ & 0 & 15 & 320 & 1280 & - & - \\
\hline Soy germ & $2 / 0$ & $0 / 0$ & 0 & 30 & 210 & - & - & - \\
\hline Spinach & $93 / 76$ & $87 / 54$ & 0 & 80 & 350 & 1440 & - & - \\
\hline
\end{tabular}




\begin{tabular}{|c|c|c|c|c|c|c|c|c|}
\hline Tomato & $326 / 367$ & $320 / 360$ & 0 & 60 & 340 & 820 & 2450 & - \\
\hline Zucchini & $149 / 93$ & $175 / 153$ & 0 & 100 & 280 & 500 & 1890 & - \\
\hline \multicolumn{9}{|l|}{ Beverages } \\
\hline Spirits & $5.5 / 32$ & $3.6 / 17$ & 0 & 80 & 300 & 560 & - & - \\
\hline Beer $\mathrm{mL}$ & $108 / 319$ & $128 / 205$ & 0 & 240 & 760 & 3750 & - & - \\
\hline Coffee mL & $250 / 267$ & $247 / 282$ & 0 & 195 & 280 & 480 & 1280 & - \\
\hline Sweet beverages $\mathrm{mL}$ & $272 / 382$ & $402 / 356$ & 0 & 1980 & 3000 & 11880 & - & - \\
\hline Tea & $1.9 / 4.4$ & $2.6 / 1.1$ & 0 & 1 & 5 & 21 & 210 & - \\
\hline Water $[\mathrm{L}]$ & $6.3 / 6.8$ & 7.1/7.3 & 0 & 2.8 & 5.8 & 8.5 & 21.0 & - \\
\hline Wine white $\mathrm{mL}$ & $25 / 93$ & $27 / 36$ & 0 & 65 & 380 & 1820 & - & - \\
\hline Wine red $\mathrm{mL}$ & $157 / 609$ & $92 / 415$ & 0 & 65 & 325 & 800 & 4400 & \\
\hline \multicolumn{9}{|c|}{ Meat, processed meat, and fish } \\
\hline White meat & $171 / 178$ & $293 / 201$ & 0 & 50 & 190 & 380 & 980 & - \\
\hline Red meat & $201 / 260$ & $209 / 216$ & 0 & 100 & 140 & 400 & - & - \\
\hline Salami & $124 / 184$ & $117 / 135$ & 0 & 75 & 170 & 280 & 340 & 820 \\
\hline Offal & $13 / 14$ & $6 / 13$ & 0 & 50 & 140 & 340 & $\cdot$ & - \\
\hline Bacon & $16 / 28$ & $24 / 12$ & 0 & 26 & 80 & 160 & - & - \\
\hline Ham & $70 / 63$ & $53 / 76$ & 0 & 30 & 120 & 250 & 700 & - \\
\hline Speck & $3 / 8$ & $6 / 8$ & 0 & 30 & 90 & 180 & - & - \\
\hline Fish & $206 / 224$ & $202 / 230$ & 0 & 70 & 140 & 700 & 1400 & - \\
\hline Canned tuna & $24 / 23$ & $19 / 53$ & 0 & 24 & 82 & 180 & 800 & - \\
\hline \multicolumn{9}{|l|}{ Dairy products } \\
\hline Milk mL & $846 / 639$ & $707 / 412$ & 0 & 400 & 900 & 1400 & 2800 & 4800 \\
\hline Cheese & $149 / 181$ & $145 / 168$ & 0 & 95 & 190 & 295 & 635 & - \\
\hline Ice cream & $38 / 41$ & $50 / 54$ & 0 & 25 & 75 & 125 & 700 & - \\
\hline Mozzarella & $70 / 67$ & $76 / 72$ & 0 & 25 & 75 & 160 & 640 & - \\
\hline Ricotta cheese & $37 / 30$ & $35 / 34$ & 0 & 25 & 70 & 120 & 490 & - \\
\hline Yogurt & $107 / 61$ & $90 / 78$ & 0 & 60 & 360 & 1680 & - & - \\
\hline Eggs & $57 / 61$ & $55 / 60$ & 0 & 25 & 125 & 350 & 700 & - \\
\hline \multicolumn{9}{|l|}{ Dressing } \\
\hline Butter & $7 / 7$ & $6 / 8$ & 0 & 40 & 160 & 270 & - & - \\
\hline Mayonnaise & $2 / 4$ & $4 / 1$ & 0 & 5 & 21 & 320 & - & - \\
\hline Margarine & $1 / 1$ & $2 / 2$ & 0 & 10 & 140 & - & - & - \\
\hline Olive oil & $108 / 105$ & $104 / 104$ & 0 & 100 & 180 & 405 & & \\
\hline \multicolumn{9}{|l|}{ Desserts } \\
\hline Homemade jam & $10 / 11$ & $13 / 12$ & 0 & 20 & 40 & 210 & - & - \\
\hline Honey & $1 / 1$ & $3 / 1$ & 0 & 3 & 35 & 85 & - & - \\
\hline Chocolate & $17 / 19$ & $17 / 9$ & 0 & 15 & 55 & 210 & - & - \\
\hline Cake & $149 / 155$ & $149 / 107$ & 0 & 35 & 175 & 560 & 1330 & - \\
\hline \multicolumn{9}{|c|}{ Other foods miscellanea } \\
\hline Sweeteners & $1.1 / 1.0$ & $0.9 / 2.2$ & 0 & 1 & 5 & 7 & 21 & - \\
\hline Salt added & $25 / 25$ & $24 / 23$ & 0 & 12 & 22 & 35 & 43 & 140 \\
\hline Sugar added & $37 / 31$ & $35 / 33$ & 0 & 14 & 31 & 75 & 270 & - \\
\hline
\end{tabular}

a example: biscuits - discretization category 1 (from 0 to $40 \mathrm{~g}$ ); category 2 (from $40 \mathrm{~g}$ to $140 \mathrm{~g}$ ); category 3 (from $140 \mathrm{~g}$ to $280 \mathrm{~g}$ ); category 4 (from $280 \mathrm{~g}$ to $480 \mathrm{~g}$ ); ${ }^{\mathrm{b}}$ Fruit of the season: mainly figs, peaches, apricot, strawberry, pear, and cherries.

was significantly lower in MetS group with a Nominal Logistic Fit value (NLF) of $\mathrm{p}=0.03804$.

-for fruits, in the female groups no significant differences were found between groups, whereas in males with MetS the intake of the fruit of the season was found significantly lower (NLF $\mathrm{p}=0.04556$ )

-for vegetables, in the females group with MetS differences were found in the carrots and cauliflowers intakes which were significantly lower with MetS (respectively an NLF with $\mathrm{p}=0.03182$ and 0.03796 ). In the males group garlic and carrots intakes were significantly lower, respectively with an NFT with $\mathrm{p}=0.01764$ and 0.04715 .

-for beverages, in the females group with MetS the red wine intake was significantly lower $(\mathrm{NLF} p=0.00232)$ and in man the red wine and beer intakes were significantly lower in the MetS group (respectively NLF $\mathrm{p}=0.03694$ and 0.04853 ).
-For meat, processed meat and fish categories, in females with MetS the white meat intake was significantly higher than Controls (NFT $\mathrm{p}=0.01515)$, whereas for all the other foods within the categories no significant differences were detected. For males the intakes of salami and offal were significantly lower in MetS group (respectively NFT $\mathrm{p}$ $=0.02932$ and 0.0414$)$, whereas in the same group the canned tuna consumption was much higher (NFT p $=0.03473)$.

- For dairy derivatives, in females no differences were found between the two groups, whereas in males the milk intake was significantly lower in the MetS subjects (NFT p $=0.04176$ ).

-In the miscellanea group, no differences between MetS and Controls were found a part of chocolate whose intake was lower in males of the MetS group (NFT p = 0.0397).

All the differences determined through the NFT were confirmed by the relative analysis of the Odds Ratios. 
Table 6. Percentages of subject in each discretization category (from D1 to D5) divided by sex

\begin{tabular}{|c|c|c|c|c|c|c|c|c|c|c|c|c|c|c|c|c|c|c|c|c|}
\hline \multirow{3}{*}{$\begin{array}{l}\text { Gender } \\
\text { Disease } \\
\text { Discretization }[\mathrm{D}]\end{array}$} & \multicolumn{10}{|c|}{ Female } & \multicolumn{10}{|c|}{ Male } \\
\hline & \multicolumn{5}{|c|}{ Controls } & \multicolumn{4}{|c|}{ MetS } & & \multicolumn{5}{|c|}{ Controls } & \multicolumn{5}{|c|}{ MetS } \\
\hline & 1 & 2 & 3 & 4 & 5 & 1 & 2 & 3 & 4 & 5 & 1 & 2 & 3 & 4 & 5 & 1 & 2 & 3 & 4 & 5 \\
\hline \multicolumn{21}{|c|}{ Carbohydrates based foods } \\
\hline Biscuits & 42 & 38 & 17 & 3 & - & 55 & 29 & 13 & 3 & - & 58 & 29 & 9 & 4 & - & 67 & 19 & 13 & 1 & $-\mathbf{a}$ \\
\hline Bread & 25 & 33 & 34 & 8 & - & 17 & 38 & 36 & 9 & - & 15 & 24 & 45 & 16 & - & 9 & 32 & 49 & 9 & - \\
\hline Breadsticks & 92 & 6 & 2 & - & - & 94 & 4 & 2 & - & - & 92 & 6 & 1 & - & - & 92 & 8 & 0 & - & - \\
\hline Croissant & 76 & 21 & 3 & - & - & 80 & 15 & 5 & - & - & 80 & 15 & 5 & - & - & 77 & 21 & 1 & - & - \\
\hline Crackers & 83 & 7 & 10 & - & - & 80 & 9 & 11 & - & - & 90 & 5 & 5 & - & - & 92 & 5 & 3 & - & - \\
\hline Gnocchi & 83 & 0 & 7 & 10 & - & 81 & 1 & 8 & 10 & - & 78 & 1 & 6 & 14 & - & 83 & 1 & 3 & 13 & - \\
\hline Oatmeal & 91 & 8 & 1 & - & - & 94 & 4 & 2 & - & - & 97 & 2 & 1 & - & - & 95 & 5 & 0 & - & - \\
\hline Rusks & 66 & 8 & 10 & 15 & - & 55 & 9 & 13 & 23 & - & 78 & 6 & 7 & 9 & - & 79 & 7 & 5 & 9 & - \\
\hline Sandwich homemade & 63 & 24 & 12 & 1 & - & 65 & 28 & 6 & 1 & - & 61 & 26 & 11 & 2 & - & 60 & 24 & 13 & 3 & - \\
\hline Sandwich commercial & 90 & 8 & 1 & - & - & 88 & 10 & 2 & - & - & 89 & 9 & 2 & - & - & 88 & 10 & 2 & - & - \\
\hline Pasta & 26 & 49 & 21 & 4 & - & 30 & 38 & 30 & 2 & - & 15 & 41 & 37 & 7 & - & 12 & 41 & 36 & 11 & - \\
\hline Polenta & 96 & 3 & 1 & - & - & 96 & 2 & 1 & - & - & 99 & 1 & 0 & - & - & 97 & 1 & 1 & - & - \\
\hline Pizza & 31 & 30 & 36 & 3 & - & 26 & 41 & 31 & 2 & - & 28 & 34 & 34 & 4 & - & 33 & 32 & 33 & 1 & - \\
\hline Potatoes & 75 & 19 & 6 & - & - & 83 & 11 & 6 & - & - & 81 & 10 & 9 & - & - & 77 & 17 & 5 & - & - \\
\hline Rice & 48 & 43 & 8 & 1 & - & 50 & 41 & 7 & 2 & - & 51 & 39 & 6 & 4 & - & 55 & 35 & 11 & 0 & - \\
\hline Tortellini & 87 & 9 & 3 & - & - & 81 & 14 & 5 & - & - & 85 & 11 & 4 & - & - & 89 & 7 & 4 & - & - \\
\hline Fruits & & & & & & & & & & & & & & & & & & & & \\
\hline Apples & 42 & 28 & 21 & 9 & - & 39 & 33 & 17 & 11 & - & 39 & 27 & 17 & 17 & - & 43 & 21 & 20 & 16 & - \\
\hline Banana & 55 & 30 & 15 & - & - & 57 & 32 & 11 & - & - & 59 & 23 & 8 & - & - & 63 & 27 & 10 & - & - \\
\hline Citrus fruit & 44 & 49 & 7 & - & - & 56 & 38 & 6 & - & - & 41 & 51 & 8 & - & - & 49 & 45 & 5 & - & - \\
\hline Dried fruit & 69 & 21 & 10 & - & - & 57 & 25 & 8 & - & - & 70 & 18 & 12 & - & - & 66 & 23 & 11 & - & - \\
\hline Fruit in syrup & 95 & 0 & 5 & - & - & 89 & 0 & 11 & - & - & 94 & 0 & 6 & - & - & 99 & 0 & 1 & - & - \\
\hline Fruit of the season ${ }^{b}$ & 62 & 18 & 12 & 8 & - & 57 & 13 & 15 & 14 & - & 60 & 16 & 15 & 9 & - & 71 & 7 & 9 & 13 & $-\mathbf{a}$ \\
\hline Fruit juice & 95 & 5 & - & - & - & 94 & 6 & - & - & - & 95 & 4 & 1 & - & - & 88 & 9 & 3 & - & - \\
\hline Grapes & 81 & 10 & 9 & - & - & 82 & 10 & 8 & - & - & 83 & 8 & 9 & - & - & 91 & 2 & 7 & - & - \\
\hline Homemade fruit juice & 88 & 7 & 5 & - & - & 86 & 8 & 6 & - & - & 89 & 7 & 4 & - & - & 83 & 9 & 8 & - & - \\
\hline Pineapple & 87 & 11 & 2 & - & - & 83 & 13 & 4 & - & - & 92 & 7 & 1 & - & - & 84 & 11 & 5 & - & - \\
\hline Plums & 98 & 2 & 0 & - & - & 96 & 4 & 0 & - & - & 99 & 0 & 1 & - & - & 99 & 1 & 0 & - & - \\
\hline Vegetables and pulses & & & & & & & & & & & & & & & & & & & & \\
\hline Carrots & 58 & 32 & 10 & - & - & 71 & 20 & 8 & - & $-\mathbf{a}$ & 63 & 27 & 10 & - & - & 70 & 27 & $\underline{3}$ & - & $-\mathbf{a}$ \\
\hline Cauliflower & 73 & 16 & 11 & - & - & 87 & 9 & $\underline{4}$ & - & $-\mathbf{a}$ & 82 & 12 & 6 & - & - & 81 & 15 & 4 & - & - \\
\hline Celery & 74 & 22 & 4 & - & - & 78 & 17 & 5 & - & - & 85 & 12 & 3 & - & - & 88 & 7 & 5 & - & - \\
\hline Chicory/lettuce & 31 & 42 & 21 & 1 & 5 & 36 & 33 & 25 & 2 & 4 & 27 & 41 & 25 & 3 & 4 & 35 & 37 & 20 & 1 & 7 \\
\hline Fennels & 92 & 8 & - & - & - & 96 & 4 & - & - & - & 92 & 8 & 0 & - & - & 95 & 4 & 1 & - & - \\
\hline Garlic & 63 & 30 & 7 & - & - & 60 & 33 & 7 & - & - & 62 & 31 & 7 & - & - & 77 & 19 & 4 & - & $-\mathbf{a}$ \\
\hline Onions & 62 & 32 & 6 & - & - & 65 & 30 & 5 & - & - & 60 & 34 & 6 & - & -- & 64 & 31 & 5 & - & - \\
\hline Pepper & 72 & 20 & 8 & - & - & 68 & 20 & 13 & - & - & 72 & 18 & 10 & - & - & 77 & 15 & 8 & - & - \\
\hline Pulses dry & 55 & 26 & 16 & 3 & - & 54 & 31 & 8 & 7 & - & 53 & 21 & 16 & 10 & - & 59 & 17 & 13 & 11 & - \\
\hline Pulses canned & 72 & 16 & 9 & 3 & - & 76 & 13 & 7 & 4 & - & 73 & 17 & 5 & 5 & - & 68 & 25 & 4 & 3 & - \\
\hline Savoy cabbage & 83 & 14 & 3 & - & - & 84 & 12 & 4 & - & - & 80 & 13 & 6 & - & - & 83 & 12 & 5 & - & - \\
\hline Soy germs & 98 & 2 & - & - & - & 99 & 1 & - & - & - & 99 & 1 & - & - & - & 99 & 1 & - & - & - \\
\hline Spinach & 69 & 23 & 8 & - & - & 69 & 27 & 4 & - & - & 74 & 20 & 6 & - & - & 85 & $\underline{8}$ & 7 & - & - \\
\hline Tomatoes & 27 & 39 & 27 & 7 & - & 37 & 31 & 19 & 13 & - & 27 & 30 & 29 & 14 & - & 27 & 36 & 26 & 11 & - \\
\hline Zucchini & 57 & 20 & 15 & 8 & - & 62 & 20 & 11 & 7 & - & 67 & 17 & 12 & 4 & - & 67 & 11 & 14 & 8 & - \\
\hline Beverages & & & & & & & & & & & & & & & & & & & & \\
\hline Spirits & 97 & 3 & - & - & - & 99 & 1 & - & - & - & 86 & 12 & 2 & - & - & 91 & 9 & 0 & - & - \\
\hline Beer & 76 & 23 & 1 & - & - & 74 & 24 & 2 & - & - & 53 & 37 & 10 & - & - & 75 & 17 & 8 & - & $-\mathbf{a}$ \\
\hline Coffee & 24 & 36 & 31 & 9 & - & 23 & 37 & 32 & 8 & - & 26 & 34 & 30 & 10 & - & 27 & 33 & 29 & 11 & - \\
\hline Sweet beverages & 97 & 2 & 1 & - & - & 94 & 5 & 1 & - & - & 93 & 4 & 3 & - & - & 91 & 6 & 3 & - & - \\
\hline Tea & 73 & 14 & 12 & 1 & - & 72 & 15 & 12 & 1 & - & 70 & 17 & 12 & 1 & - & 71 & 16 & 12 & 1 & - \\
\hline Water & 3 & 32 & 40 & 25 & - & 2 & 33 & 39 & 26 & - & 2 & 35 & 37 & 26 & - & 3 & 31 & 41 & 25 & - \\
\hline Wine white & 86 & 12 & 2 & - & - & 86 & 13 & 1 & - & - & 81 & 13 & 6 & - & - & 90 & 7 & 3 & - & - \\
\hline Wine red & 61 & 19 & 15 & 5 & - & 73 & 19 & 3 & 5 & $-\mathbf{a}$ & 20 & 17 & 29 & 34 & - & 45 & 20 & 16 & 19 & $-\mathbf{a}$ \\
\hline Meat, processed meat & & & & & & & & & & & & & & & & & & & & \\
\hline White meat & 26 & 28 & 35 & 8 & 3 & 12 & 36 & 32 & 12 & $8 \mathbf{a}$ & 25 & 28 & 34 & 9 & 4 & 28 & 23 & 29 & 11 & 9 \\
\hline Red meat & 69 & 22 & 9 & - & - & 80 & 12 & 8 & - & - & 74 & 13 & 13 & - & - & 77 & 16 & 7 & - & - \\
\hline Salami & 48 & 17 & 20 & 5 & 10 & 54 & 13 & 16 & 9 & 8 & 38 & 13 & 21 & 9 & 19 & 37 & 29 & 17 & 8 & $8 \mathbf{a}$ \\
\hline
\end{tabular}




\begin{tabular}{|c|c|c|c|c|c|c|c|c|c|c|c|c|c|c|c|c|c|c|c|c|}
\hline Offal & 93 & 2 & 5 & - & - & 95 & 1 & 4 & - & - & 89 & 6 & 5 & - & - & 95 & 0 & 5 & - & $-\mathbf{a}$ \\
\hline Bacon & 76 & 15 & 9 & - & - & 79 & 15 & 6 & - & - & 75 & 15 & 10 & - & - & 74 & 16 & 10 & - & - \\
\hline Ham & 38 & 33 & 27 & 2 & - & 45 & 32 & 21 & 1 & - & 41 & 27 & 28 & 4 & - & 37 & 28 & 29 & 5 & - \\
\hline Speck & 95 & 3 & 1 & - & - & 92 & 6 & 2 & - & - & 86 & 10 & 4 & - & - & 89 & 8 & 3 & - & - \\
\hline Fish & 29 & 59 & 11 & 1 & - & 35 & 52 & 12 & 1 & - & 31 & 52 & 12 & 5 & - & 27 & 60 & 11 & 2 & - \\
\hline Canned tuna & 76 & 17 & 6 & 1 & - & 79 & 15 & 6 & 0 & - & 81 & 12 & 4 & 3 & - & 67 & 17 & 9 & 7 & $-\mathbf{a}$ \\
\hline \multicolumn{21}{|l|}{ Dairy products } \\
\hline Milk & 38 & 17 & 14 & 8 & - & $\underline{51}$ & 11 & 8 & 25 & 5 & 54 & 10 & 8 & 22 & 5 & 60 & 19 & 8 & 13 & $0 \mathbf{a}$ \\
\hline Cheese & 32 & 35 & 21 & 12 & - & 31 & 43 & 13 & 13 & - & 26 & 29 & 27 & 18 & - & 36 & 27 & 17 & 20 & - \\
\hline Ice cream & 64 & 15 & 12 & 9 & - & 62 & 12 & 11 & 15 & - & 68 & 11 & 11 & 10 & - & 57 & 13 & 15 & 15 & - \\
\hline Mozzarella & 39 & 23 & 28 & 10 & - & 36 & 25 & 25 & 14 & - & 41 & 22 & 27 & 10 & - & 41 & 19 & 28 & 12 & - \\
\hline Ricotta cheese & 60 & 17 & 14 & 9 & - & 62 & 18 & 14 & 6 & - & 57 & 22 & 13 & 8 & - & 63 & 15 & 14 & 8 & - \\
\hline Yogurt & 68 & 18 & 14 & - & - & 71 & 17 & 12 & - & - & 80 & 9 & 11 & - & - & 83 & 8 & 9 & - & - \\
\hline Eggs & 40 & 47 & 12 & 1 & - & 45 & 43 & 11 & 1 & - & 38 & 43 & 17 & 2 & - & 40 & 44 & 15 & 1 & - \\
\hline \multicolumn{21}{|l|}{ Dressing } \\
\hline Butter & 93 & 7 & - & - & - & 96 & 4 & - & - & - & 94 & 5 & 1 & - & - & 92 & 8 & 0 & - & - \\
\hline Mayonnaise & 89 & 7 & 4 & - & - & 84 & 12 & 4 & - & - & 88 & 10 & 23 & - & - & 91 & 8 & 1 & - & - \\
\hline Margarine & 96 & 4 & - & - & - & 96 & 4 & - & - & - & 99 & 1 & - & - & - & 98 & 2 & - & - & - \\
\hline Olive oil & 40 & 43 & 17 & - & - & 35 & 51 & 13 & - & - & 36 & 51 & 13 & - & - & $\underline{48}$ & $\underline{30}$ & 21 & - & - \\
\hline \multicolumn{21}{|l|}{ Desserts } \\
\hline Homemade jam & 81 & 9 & 10 & - & - & 80 & 4 & 14 & - & - & 78 & 9 & 13 & - & - & 79 & 10 & 11 & - & - \\
\hline Honey & 85 & 7 & 8 & - & - & 84 & 6 & 10 & - & - & 85 & 6 & 9 & - & - & 86 & 5 & 5 & - & - \\
\hline Chocolate & 62 & 29 & 9 & - & - & 63 & 28 & 9 & - & - & 64 & 27 & 9 & - & - & 82 & 8 & 10 & - & $-\mathbf{a}$ \\
\hline Cake & 35 & 31 & 28 & 6 & - & 37 & 29 & 29 & 5 & - & 36 & 30 & 27 & 7 & - & 35 & 30 & 29 & 6 & - \\
\hline \multicolumn{21}{|c|}{ Other foods miscellanea and water } \\
\hline Sweeteners & 83 & 8 & 2 & 7 & - & 80 & 6 & 4 & 10 & - & 82 & 9 & 3 & 6 & - & 81 & 10 & 4 & 5 & - \\
\hline Salt & 13 & 46 & 16 & 21 & 4 & 14 & 45 & 18 & 17 & 6 & 12 & 47 & 16 & 20 & 5 & 13 & 47 & 15 & 20 & 5 \\
\hline Sugar (added) & 31 & 15 & 42 & 11 & - & 40 & 27 & 30 & 13 & - & 30 & 16 & 40 & 14 & - & 35 & 18 & 27 & 20 & - \\
\hline
\end{tabular}

$\mathbf{a}=$ Nominal Logistic Fit $\mathrm{p}<0.05$ Controls Vs MetS; see Table 5 for the average values

Table 7. Likelyhood ratio test for genders: foods within the observed range (whole model test)

\begin{tabular}{|c|c|c|c|}
\hline \multicolumn{2}{|c|}{ Females } & \multicolumn{2}{|c|}{ Males } \\
\hline variable increase & pertinence to & variable increase & pertinence to \\
\hline Water & MetS & Carrot & Controls \\
\hline Alcohol & Controls & Chicory & MetS \\
\hline Butter & Controls & Chocolate $^{\mathrm{a}}$ & Controls \\
\hline Coffee & MetS & Dried fruit & MetS \\
\hline Crackers & MetS & Fennels & MetS \\
\hline Fruit syrup & MetS & Fruit of the season & MetS \\
\hline Fruit of the season & MetS & Garlic $^{\text {a }}$ & Controls \\
\hline Ham & Controls & Ham & MetS \\
\hline Homemade jam & MetS & Homemade jam & MetS \\
\hline Homemade sandwich & Controls & Ice cream & MetS \\
\hline Homemade fruit juice & MetS & Milk $^{a}$ & Controls \\
\hline Lettuce & Controls & Pasta & MetS \\
\hline Mayonnaise & MetS & Red Wine $^{\text {a }}$ & Controls \\
\hline Potato & Controls & Sweeteners & MetS \\
\hline Onions & Controls & White wine & Controls \\
\hline \multirow[t]{3}{*}{ White meat ${ }^{\mathrm{a}}$} & Mets & Yogurt & Controls \\
\hline & & Zucchini & MetS \\
\hline & & Pinapple & MetS \\
\hline
\end{tabular}

$\mathrm{a}=$ foods that were shown to be significantly different comparing the control Vs MetS if tested separately

The combination of foods that can be characteristic of MetS and Controls were reported according to the Likelihood Ratio Test (Table 7).

It is evident that considering the combination of foods, a very different picture is coming out, such that only two foods (fruit of the season and homemade Jam) are common for the two sexes and all the other are different and a more complex figure seems to takes shape in the development of the MetS or to maintain the control condition.
Some aspects are very difficult to explain as for example the increase of the fruit of the season that turns out to increase the possibility of MetS in both sexes, whereas in the single item analysis a non-significant higher intake was found in females only.

\section{Discussion}

Some limitations are present in this research concerning first of all the enrollment, since a selection was operated by the doctors asking their patients with MetS to participate matching them subjects of the 
same age. This means that only subjects interested to the relationship between food and MetS were participating to the study.

The second weak point concerns the foods tested because they were representing about $90 \%$ of the total amount of the weekly caloric intake, and the lowest percentages were represented in the two groups suffering from MetS. This means that theoretically about $10 \%$ of the foods not included in the analysis could be partially responsible of MetS.

Another limitation can be determined by the lack of evaluation of micronutrients that are considered to counteract MetS [20,21].

However, the micronutrients that were not part of the analysis were in relation only to the about $10 \%$ of foods not considered in the analysis and should not influence critically the comparison between groups.

In general, the subjects participating to this study were those that the family doctor has to take care off in the daily practice and most of Control cases could not considered belonging to a healthy population.

Furthermore, it was not possible to compare the data of the three countries because the relative numbers were not enough to compare the two groups of subjects.

A part of these limitations some interesting indications can be drawn from the study.

The first observation which was common for both, female and males suffering from MetS, was a more consistent caloric intake. Compared to the values calculated with MSJ algorithm the caloric intake in MetS subjects was respectively $>9.5 \%$ in females and $>4.7 \%$ in males. Despite not excessive, at long term these increases may be determinant for the development of the disease.

The age of the two groups was $<52$ years and one may not speculate about the incidence of MetS in relation to age since the subjects were selected by the family doctors. Because of this the incidence of the disease in the present study are higher than what has been determined in a previous survey conducted in some Italian towns where the incidence in a population between 35 and 65 years was $<20 \%$ of the cases [16].

Foods were found to have a different impact in relation to the gender, and this could mean that the epidemiological studies should consider these aspects in long term survey and also the need of different treatment in terms of drugs.

The reason of this difference between genders is unknown and probably belongs to the hormonal balance. This hypothesis may arise also by the evidence that the intake of white meat was more consistent in females with MetS. The quite common practice (although is prohibited) to give estrogens to reduce the aggression of chickens and turkey in breeding farm may be one of the causes. Meat deriving from these animals is a substantial part of the white meat intake in Italy.

Some data that are considered common determinants of MetS, such as the excessive intake of carbohydrates, is not emerging from this study. At the opposite the total amount of carbohydrate-based food (consisting of about $80 \%$ of the weight in carbohydrates) were respectively $1366 \mathrm{~g}$ in the normal females and $1203 \mathrm{~g}$ in females with MetS, the same was for males consisting of $1606 \mathrm{~g} /$ week for controls and $1499 \mathrm{~g} /$ week for MetS.
The amount of calories from fats were similar in all the groups (difference $<2 \%$ ) and also the ratio between carbohydrates or soluble sugars and fats was not having a discriminant power to differentiate MetS.

Some author found that carbohydrate restriction has a more favorable impact on the disease than low fat diet and our results do not confirm these findings [22]. Furthermore, our data do not confirm the negative impact of soluble sugars as other author have shown to be associated with MetS in females [23].

In terms of processed meat, it was shown that salami intake was lower in the MetS group and also the offal intake.

This may indicate more prudence in defining the processed meat as "carcinogenic to humans" [24].

A part of the quantity of processed meat intake, a quite careful distinction should be made about the way of producing this type of food which in many cases do not contain nitrates (or minimal amount), particularly when they are produced directly by the consumers like in some rural community.

The red wine seems to be protective in males, at least in the amount used in this study as average $610 \mathrm{~mL} /$ week in the Control group and 415 $\mathrm{mL} /$ week in the MetS group, that at the end was less than one alcoholic unit/day for both groups.

Other foods such as garlic and chocolate seem to be protective in males (not in females) and one may speculate the presence of powerful antioxidants in both $[25,26]$.

One particular food, canned tuna, was found significantly more used in MetS males. Fish and fish oil are giving conflictual results on the disease, but recently were considered healthy for some author [27]. In our study we could not show any favorable activity of these foods. This is an example on how the food quantities, the context of a diet, the country, down to the single community may be determinant for the disease. All these aspect makes more complex the pathogenesis of MetS which also belongs to the genetics and epigenetics traits together with the microbiota.

Once the foods were analyzed together, a quite different picture was emerging where the interactions take place and single elements are diluted or amplified such that even drinking water may have a role (in females only), and genders differences are becoming extremely evident. Among the 17 foods for females and 18 foods for males which are bound respectively to the Controls or MetS conditions, only two are common: fruit of the season and homemade jam whose increasing intake was found related to the MetS.

$\mathrm{Al}$ the other foods are different, no matter if the Increased quantities are bound to MetS or Controls.

One further important aspect arising from this study has to be mentioned and concerns the concomitant diseases. It was clear that people suffering from MetS have to be treated with more drugs. Considering the costs in terms of drug expenses- which in Italy is covered mostly by the Government- for an average life expectancy of 25 years more (from about 50 years up to about 75 ) the estimated cost is $>€ 20,000 /$ subject.

This means that will be much more convenient to use this money for prevention and education to give more life to the years. 


\section{Conclusions}

In the context of a Mediterranean diet and in our experimental conditions it seems that MetS both in females and males was determined by higher amount of caloric intake, without a clear distinction between carbohydrates, fats, fibers, proteins and alcohol. Concomitant diseases such as CVD and osteoarthritis (in males only) were more common indicating a fragile healthy condition in subjects suffering from MetS.

A part of the caloric intake, the pattern of foods bound to the MetS was found completely different in females and males.

Some particular foods were more common in MetS, such as white meat (in females) or seasonal fruits and homemade fruit juice (for both genders). In males, the processed meat and red wine intake -in the relative limited quantities that have been determined - were found to be protective.

However, once the different foods were analyzed together a completely different relationship with MetS emerged, indicating that a single food category cannot be considered the cause of the disease which seems to be determined by the interactions of many foods, that again are different for females and males.

The disease has a heavy social and economic impact and despite common diagnostic aspects the causes and the remedies of MetS in terms of foods have such a proteiform aspect that any community (country, town, village) could have peculiar way to face it.

Larger epidemiological studies in each territorial context with specific food pattern are needed to give appropriate information on healthy foods. These aspects belong to the social/educational area and can be faced only with the help of the Governments, family doctors and local Municipalities.

\section{Acknowledgements}

The study was supported largely by private funding and no competing interest have to be declared. The Bracco Spa- Milan Italy was partially supporting this study with a grant of $€ 100,000$.

The authors are grateful to the Municipalities of Rende, Rovito and San Lucido which made the surgeries available.

\section{Contribution}

UC was preparing the protocol, visited all the subjects and wrote the article; GC visited all the subjects and was charged for the FIA; GM was charged with the ultrasound analysis; MR was assisting in the logistic of the investigation; OV was taking care of data input; MR was making all the statistical calculations using JMP Pro 14.1 software of SAS institute Inc.

\section{References}

1. Eckel SM, Alberti KG, Grundy SM (2010) The metabolic syndrome. Lancet 375: 181183.

2. O'Neil S, O'Driscoll L (2015) Metabolic syndrome: a closer look at the growing epidemic and its associated pathologies. Obesity rev 16: 1-12. [Crossref]

3. Samson SL, Gaber AJ (2014) Metabolic syndrome. Endocrinol Metab Clin North Am 43: 1-23.

4. Bellia A, Giardina E, Lauro D (2009) The Linosa study: epidemiological and heritability data of the metabolic syndrome in a Caucasian genetic isolate. Nutr Metab Cardiovasc Dis 19: 455-461. [Crossref]

5. Kissebah AH, Sonnenberg GE, Myclebust J (2000) Quantitative trait loci in chromosomes 3 and 17 influence phenotypes of the metabolic syndrome. Proc Natl Acad Sci USA 97: 14478-14483. [Crossref]
6. Loss RJ, KattamarzyK PT, Rao DC (2003) Genome-wide linkage scan for the metabolic syndrome in the HERITAGE Family Study. J Clin Endocrinol Metabol 88: 5935-5943. [Crossref]

7. Edwards KL, Hutter CM, Wan JY (2008) Genome-wide linkage scan for metabolic syndrome: the GENNID study. Obesity (Silver Spring) 16: 1596-1601. [Crossref]

8. Fryling TM, Timpson M, Weedon MN (2007) A common variant in the FTO gene is associated with body mass index and predispones to childhood and adult obesity. Science 316: 889-894. [Crossref]

9. Torres S, Fabersani E, Marquez A (2018) Adipose tissue inflammation and metabolic syndrome. The proactive role of probiotics. Eur J Nutr. [Crossref]

10. Festi D, Schiumerini R, Eusebi LH (2014) Gut microbiota and metabolic syndrome. World J Gastroenterol 20: 16079-16094. [Crossref]

11. Kaur JA (2014) Comprehensive review on metabolic syndrome. Cardiol Res Pract 2014: 941162. [Crossref]

12. Ludwig DS (2018) Dietary carbohydrates: role of quality and quantity in chronic disease. $B M J$ [Crossref]

13. Yamaoka K, Tango T (2012) Effects of lifestyle modification on metabolic syndrome: a systematic review and metanalysis. BMC Med 10: 138. [Crossref]

14. Kastorini CM, Milions HJ, Esposito K (2011) The effect of Mediterranean diet on metabolic syndrome and its component. J Am Coll Cardiology 57: 1299-1313. [Crossref]

15. Babio N, Toledo E, Estruch R (2014) Mediterranean diets and metabolic syndrome status in the PREDIMED randomized trial. CMAJ 186: E649-E657. [Crossref]

16. Cornelli U, Milani L, Perra A (2006) The use of polyglucosamine in the metabolic syndrome control. Med Biol 4: 45-54.

17. Cornelli U, Belcaro G, Recchia M, D'Orazio N (2017) Long-term treatment of overweight and obesity with polyglucosamine (PG L112): randomized study compared with placebo in subjects after caloric restriction. Curr Dev Nutr. [Crossref]

18. Belcaro G, Nicolaides AN, Laurora G (1996) Ultrasound morphology classification of the arterial wall and cardiovascular events in a 6-year follow-up study. Arterioscl Thromb Vasc Biol 16: 851-856. [Crossref]

19. Lesaffre E, Albert A (1989) Multiple group logistic regression diagnostic. Appl Stat 38: 425-440.

20. Goncalves A, Amiot MJ (2017) Fat-soluble micronutrients and metabolic syndrome Curr Opin Clin Nutr Metab care 26: 492-497. [Crossref]

21.

22. Dakshinamurti K (2015) Vitamins and their derivatives in the prevention and treatmen of metabolic syndrome disease (diabetes). Can J Physiol Pharmacol 93: 355-362. [Crossref]

23. Volek JS, Phinney SD, Forsythe CE (2009) Carbohydrate restriction has more favorable impact on the metabolic syndrome than low fat diet. Lipids 44: 297-309. [Crossref]

24. Song S, Lee JE, Song WO (2014) Carbohydrate intake and refined-grain consumption are associated with metabolic syndrome in the Korean adult population. $J$ Acad Nutr Diet 114: 54-62. [Crossref]

25. Bouvard V (2015) IARC Carcinogenicity of consumption of red and processed meat Lancet oncology 16: 1599-1600. [Crossref]

26. Banerjee SK, Mukherjee PK, Maulik SK (2013) Garlic as an antioxidant: the good, the bad and the ugly. Physiother Res 17: 97-106. [Crossref]

27. Serafini M, Bugianesi R, Maiani G (2003) Plasma antioxidant from chocolate. Nature 424: 10-13. [Crossref]

28. Karlsson T, Rosendahl-Riise H,Dierkes J (2017) Association between fish intake and the metabolic syndrome and its component among middle-aged men and women: the Horderland Healthy Study. Food Nutr Res 61: 1347-1479. [Crossref]

Copyright: (C)2018 Cornelli U. This is an open-access article distributed under the terms of the Creative Commons Attribution License, which permits unrestricted use, distribution, and reproduction in any medium, provided the original author and source are credited. 